\title{
Genetics of fasting indices of glucose homeostasis using GWIS unravels tight relationships with inflammatory markers
}

Running title:

\section{HOMA-B/HOMA-IR GWIS}

Iryna O. Fedko, PhD, ${ }^{1,2, *}$ Michel G. Nivard, PhD,1,2 Jouke-Jan Hottenga, PhD, ${ }^{1,2}$ Liudmila Zudina, ${ }^{13}$ Cross Consortia Pleiotropy (XC-Pleiotropy) Group, CHARGE Inflammation working group, Zhanna Balkhiyarova, $\mathrm{PhD},{ }^{13}$ Daniel I. Chasman, $\mathrm{PhD},{ }^{3}$ Santhi Ganesh, PhD, ${ }^{4,5}$ Jie Huang, PhD, ${ }^{6,7}$ Mike A. Nalls, PhD, ${ }^{8}$ Christopher J. O’Donnell, PhD, ${ }^{9,10}$ Guillaume Paré, MD,11 Paul M. Ridker, PhD, ${ }^{3}$ Meta-Analyses of Glucose and Insulin-related traits Consortium (MAGIC) Investigators, Reedik Mägi, PhD, ${ }^{12}$ Marika Kaakinen, PhD, ${ }^{13,14}$ Inga Prokopenko, $\mathrm{PhD},{ }^{13, *, \#}$ and Dorret I. Boomsma, $\mathrm{PhD}{ }^{1,2, \#}$

${ }^{1}$ Department of Biological Psychology, Vrije Universiteit Amsterdam, The Netherlands ${ }^{2}$ Amsterdam Public Health research institute, The Netherlands

${ }^{3}$ Center for Cardiovascular Disease Prevention, Division of Preventive Medicine, Brigham and Women's Hospital, Harvard Medical School, Boston Ma USA

${ }^{4}$ Division of Cardiovascular Medicine, Department of Internal Medicine, University of Michigan Health System, Ann Arbor, MI, USA.

${ }^{5}$ Department of Human Genetics, Ann Arbor, MI, USA.

${ }^{6}$ Boston VA Research Institute, Boston, Massachusetts, USA

${ }^{7}$ Brigham Women's Hospital Division of Aging, Harvard Medical School, Boston, Massachusetts, USA 
${ }^{8}$ CEO/Consultant with Data Tecnica International, Glen Echo, MD, USA; Laboratory of Neurogenetics, National Institute on Aging, Bethesda, USA

${ }^{9}$ Cardiology Section, Department of Medicine, Boston Veterans Administration Healthcare System, Boston MA

10Department of Medicine, Brigham and Women's Hospital, Harvard Medical School, Boston MA

11Department of Pathology and Molecular Medicine, McMaster University, Hamilton, Canada

12Estonian Genome Center, Univeristy of Tartu, Tartu, Estonia

${ }^{13}$ Section of Genomics of Common Disease, Department of Medicine, Imperial College London, London, UK

${ }^{14}$ Pharmacology and Therapeutics, Department of Medicine, Imperial College London, London, UK

* Corresponding authors:

Iryna 0. Fedko, Department of Biological Psychology, Vrije Universiteit Amsterdam, Van der Boechorststraat 7-9, 1081BT, Amsterdam, The Netherlands, e-mail: i.o.fedko@vu.nl

Inga Prokopenko, Section of Genomics of Common Disease, Department of Medicine, Imperial College London, London W12 0NN, UK,

e-mail: i.prokopenko@imperial.ac.uk

\# These authors contributed equally to this work 


\begin{abstract}
Purpose: Homeostasis Model Assessment of $\beta$-cell function and Insulin Resistance (HOMA-B/-IR) indices are informative about the pathophysiological processes underlying type 2 diabetes (T2D). Data on both fasting glucose and insulin levels are required to calculate HOMA-B/-IR, leading to underpowered Genome-Wide Association studies (GWAS) of these traits.
\end{abstract}

Methods: We overcame such power loss issues by implementing Genome-Wide Inferred Statistics (GWIS) approach and subsequent dense genome-wide imputation of HOMA-B/IR summary statistics with SS-imp to 1000 Genomes project variant density, reaching an analytical sample size of 75,240 European individuals without diabetes. We dissected mechanistic heterogeneity of glycaemic trait/T2D loci effects on HOMA-B/-IR and their relationships with 36 inflammatory and cardiometabolic phenotypes.

Results: We identified one/three novel HOMA-B (FOXA2)/HOMA-IR (LYPLAL1, PER4, PPP1R3B) loci. We detected novel strong genetic correlations between HOMA-IR/-B and Plasminogen Activator Inhibitor 1 (PAI-1, $r_{g}=0.92 / 0.78, \mathrm{P}=2.13 \times 10^{-4} / 2.54 \times 10^{-3}$ ). HOMAIR/-B were also correlated with C-Reactive Protein $\left(r_{g}=0.33 / 0.28, \mathrm{P}=4.67 \times 10^{-3} / 3.65 \times 10^{-}\right.$ 3). HOMA-IR was additionally correlated with T2D $\left(r_{g}=0.56, \mathrm{P}=2.31 \times 10^{-9}\right)$, glycated haemoglobin $\left(r_{g}=0.28, \mathrm{P}=0.024\right)$ and adiponectin $\left(r_{g}=-0.30, \mathrm{P}=0.012\right)$.

Conclusion: Using innovative GWIS approach for composite phenotypes we report novel evidence for genetic relationships between fasting indices of insulin resistance/beta-cell function and inflammatory markers, providing further support for the role of inflammation in T2D pathogenesis.

Keywords: HOMA-IR, HOMA-B, type 2 diabetes, inflammation, genome-wide inferred statistics 


\section{Introduction}

Type 2 diabetes (T2D) represents the fastest growing non-communicable disease epidemic world-wide ${ }^{1}$. Fasting hyperglycaemia is one of the criteria used for type 2 diabetes (T2D) diagnosis, and fasting insulin (FI) levels are indicative of insulin sensitivity in peripheral tissues, but neither measure provides mechanistic insights into insulin secretion and action ${ }^{2}$. Homeostasis Model Assessment of $\beta$-cell function (HOMAB) and Insulin Resistance (HOMA-IR) can be derived from FG and FI concentrations and are two commonly used fasting state glycaemic indices elucidating pathophysiological processes in T2D-11. HOMA-B reflects the function of $\beta$-cells in terms of their ability to secrete insulin, whereas HOMA-IR is a surrogate measure of insulin sensitivity.

Pathophysiology of T2D and metabolic syndrome, as well as epidemiological and genetic studies suggest that there is a shared aetiology between cardiometabolic phenotypes, variability of glycaemic traits in individuals without diabetes, and $\mathrm{T}_{2} \mathrm{D}^{9,12-14}$. Sedentary lifestyle and major risk factors of T2D induce low-grade inflammation, which consequently leads to overt diabetes in individuals with more pronounced and prolonged inflammatory response $\mathrm{e}^{15}$ and insulin resistance. However, genetic relationships between these traits are not established. Moreover, metabolic syndrome is a pro-thrombotic state due to the inhibition of the fibrinolytic pathway, another proposed risk factor of T2D related to insulin resistance and inflammation ${ }^{16,17}$, but little is known about their shared genetic risk factors. The shared genetic aetiology within cluster of cardiometabolic traits was only recently reported through estimation of genetic correlations ${ }^{18,19}$.

Our understanding of biological processes shared between $\beta$-cell function and insulin resistance, reflected by HOMA-B/-IR, and a range of epidemiologically related traits and diseases, such as T2D, could be informed by dissecting the patterns of their genetic relationships. Genome-wide association studies (GWAS) have identified over 70 
loci associated with fasting glucose (FG) and/or FI levels in non-diabetic European descent individuals 20 . However, to date, only ten HOMA-B loci, including $A D C Y 5$, ARAP1(STARD10), DGKB, FADS1, GLIS3, GCK, G6PC2, MTNR1B, TCF7L2, and SLC30A8, and three HOMA-IR loci (GCKR, IGF1/SC4MOL, TCERG1L) have been described in GWA studies in European/African American descent populations ${ }^{21-25}$. This is mostly attributable to underpowered studies, since HOMA-B and HOMA-IR calculations require assessment of both FG and FI in the same individual. If either of these two primary fasting measures is missing for an individual, their HOMA indices cannot be derived and such individuals do not contribute to GWAS of HOMA indices. Similarly, missing FG or FI measurement in an entire cohort precludes involvement of such study in GWAS meta-analyses of HOMA indices (Figure 1). As a consequence, HOMA-B/-IR GWAS meta-analyses usually feature dramatically smaller sample sizes compared to FG/FI GWAS. Previously published HOMA-B/-IR discovery analyses by the Meta-Analyses of Glucose and Insulin-related traits Consortium (MAGIC) suffered from about 20\% sample size and power losses for analytically inferred indices ${ }^{21}$, which has held up the progress of understanding the molecular mechanisms behind insulin secretion and sensitivity composite measures through locus discovery in recent years 26,$27 ; 28,29$. The Genome Wide Inferred Statistics (GWIS) method provides an approximation of the GWA summary statistics for any derived variable that is a function of primary phenotypes, when such statistics, means and covariances of the constituent primary phenotypes are available or can be approximated with reasonable precision ${ }^{30}$. Unlike GWAS, the GWIS can accommodate information from individuals or cohorts, where any of the primary phenotypes is assessed. Moreover, the GWIS accommodates any degree of overlap between individuals from studies contributing data for primary traits. In this study, we applied GWIS methodology ${ }^{30}$ and derived the summary statistics for HOMA indices based on recent FG 
and FI GWAS meta-analysis summary statistics from the MAGIC (Lagou et al., manuscript in preparation).

The aims of this study were three-fold: (i) to infer analytically HOMA-B/-IR GWIS based on the FG/FI GWAS meta-analysis summary statistics from the MAGIC consortium and evaluate the sensitivity of GWIS methodology; (ii) to use the obtained summary statistics to define the effects of T2D, FG/FI and $\mathrm{HbA1}$ c loci on insulin secretion and action through their effects on HOMA-B/-IR; and (iii) to explore the genetic relationships between HOMA-B/-IR, cardiometabolic, and inflammatory traits.

\section{Methods}

Phenotypes

Homeostasis Model Assessment of $\beta$-cell function (HOMA-B) and Insulin resistance (HOMA-IR) are calculated from the Fasting Glucose and Fasting Insulin measures by formulae:

$$
H O M A-B=\frac{20 \times F I}{F G-3.5},[1] \quad H O M A-I R=\frac{F G \times F I}{22.5},[2]
$$

where $\mathrm{FG}$ is measured in $\mathrm{mmol} / \mathrm{l}$ and $\mathrm{FI}$ is in $\mathrm{mU} / \mathrm{l}$ units ${ }^{31}$.

\section{GWIS for HOMA-B/-IR}

To approximate the HOMA-B/-IR GWAS summary statistics, we applied a recently developed GWIS approach ${ }^{30}$. We obtained the summary statistics from the latest GWAS meta-analysis of FG and FI performed by the MAGIC in up to 88,320/64,090 individuals and 40/33 studies respectively (Supplementary Materials and Methods) (Lagou et al., manuscript in preparation). Studies genotyped on Metabochip were not included, so that inferred summary statistics were appropriate to analyse with LD score regression 
(LDSC) ${ }^{32}$. In the MAGIC meta-analysis FI was measured in pmol/l and natural log transformed, FG was measured in mmol/l with a cut-off at $7 \mathrm{mmol} / \mathrm{l}$. The standard HOMA formulae require untransformed FG/FI measures and use mU/l units for FI. We adapted the HOMA formulae to compute a GWAS summary statistics for $\ln$ (HOMA-IR) and $\ln ($ HOMA-B) given summary statistics for FG and $\ln (\mathrm{FI})$, measured in the units described above:

$$
H O M A-B=\ln \frac{20 \times \frac{e^{F I}}{6.945}}{F G-3.5},[3] \quad H O M A-I R=\ln \frac{F G \times \frac{e^{F I}}{6.945}}{22.5}
$$

where division by 6.945 was introduced to convert FI from pmol/l to $\mathrm{mU} / \mathrm{l}$ units. The GWIS method requires, in addition to genome wide summary statistics for FG and FI, the population mean of FG and FI, the phenotypic correlation between FG and FI and sample overlap across the studies, included in both meta-analyses studies, to correct for dependence between the FG and FI GWAS (details in Supplemental Materials and Methods and example scripts in Data Access). Overall, we calculated the effect estimates $\left(\beta^{\prime}\right.$ s) for HOMA-B and HOMA-IR for each $\mathrm{SNP}_{i, i} i=1, \ldots, \mathrm{M}$, where $\mathrm{M}$ is the number of SNPs. According to the GWIS method $\beta_{\mathrm{i}}$ is computed as a sum of HOMA-B or HOMA-IR functions respectively (versions of the formulae given above) derived using population means and published summary statistics for FG and FI, which are evaluated for 1 and 2 copies of effect alleles and weighted by the estimated frequencies of effect alleles in FI and FG GWAS summary statistics (Supplementary Materials and Methods). For the calculation of the standard errors (SEs) of the GWIS-inferred effect estimates of HOMA-B and HOMAIR we used the Delta method accounting for the sample overlap (Supplementary Materials and Methods). We compared the magnitude and direction of the effects in genome-wide significant loci in published and inferred analysis and confirmed power 
gain, especially for HOMA-IR, when compared to previous analysis. (Tables S1 and S2,

\section{Figures S1-S4).}

We run the LDSC $^{33}$ between the GWIS-inferred and published summary statistics for HOMA-B and HOMA-IR, to obtain the LDSC intercept for the GWIS-inferred HOMA-B/-IR and to estimate the genetic correlation between inferred and published data. We used LDSC intercept to correct the inferred summary statistics for any inflation (Supplementary Materials and Methods).

\section{Genome-wide imputation using summary statistics}

We implemented a novel methodology developed within SS-imp software tool that enables genome-wide imputation from denser reference panels ${ }^{34}$ and imputed the analytically inferred HOMA-B/-IR GWIS to 1000 Genomes project variant density and compared the findings with the lower variant density directly inferred GWIS.

\section{Functional and regulatory elements enrichment analysis}

We applied the GARFIELD ${ }^{35}$ tool v2 on the meta-analysis results to assess enrichment of the HOMA-B/HOMA-IR associated variants within functional and regulatory features. GARFIELD enables checking for genic annotations, chromatin states, DNaseI hypersensitive sites, transcription factor (TF) binding sites, FAIRE-seq elements and histone modifications, among others, in a number of publicly available cell lines. We considered significant enrichment to be present if the GWIS signal and the functional annotation signal significantly co-localized, i.e. $P_{\mathrm{GWIS}}<5 \times 10^{-8}$ and $P_{\text {enrichment }}<1.2 \times 10^{-5}$ after correction for 2,040 annotations. 
To evaluate the shared genetic aetiology, we applied the LDSC approach ${ }^{33}$ to HOMA-B/IR and publicly available meta-analysis summary statistics for T2D, 13 glycaemic and 17 cardiometabolic phenotypes (sample size, ethnicity, reference and source of the data presented in Table S3). We defined an extended group of cardiometabolic phenotypes and related traits by including chronic kidney disease (CKD) and its markers (Estimated glomerular filtration rate (eGFR) based on creatinine and cystatin $\mathrm{C})^{36}$; systolic and diastolic blood pressure (SBP/DBP) and hypertension (HTN) ${ }^{37}$ to explore the genetic relationships between cardiometabolic traits and HOMA-B/-IR. We expanded our analysis to five inflammatory markers, including adiponectin 38 , plasminogen activator inhibitor 1 (PAI-1) ${ }^{39}$, C-reactive protein (CRP) ${ }^{40}$, intercellular adhesion molecule 1 $(\mathrm{ICAM}-1)^{41}$, white blood cell counts $\left.(\mathrm{WBC})^{42}\right)$. The five inflammation phenotypes were obtained with permission of Cross Consortia Pleiotropy (XC-Pleiotropy) Group (Table S3).

\section{Results}

GWAS for HOMA-B/-IR

We inferred analytically the HOMA-B/-IR GWAS summary statistics using the summary statistics of HapMap reference panel-imputed FG/FI GWAS meta-analyses of 40/33 studies by the MAGIC (Lagou et al., in preparation). We imputed them to 1000 Genomes phase 3 (2013-05-02) reference panel thus reaching $\sim 11.2 \mathrm{M}$ autosomal variants for up to 75,240 non-diabetic individuals of European descent. We detected a novel HOMA-B locus at FOXA2 gene (rs5029909, $\beta(\mathrm{SE})=-0.044(0.0079), \quad \mathrm{P}=2.70 \times 10^{-08}$ ), which overlapped with an established FG-association (rs6113722, $\mathrm{R}^{2}=0.69$ in Europeans, 1000G) ${ }^{13}$. We also confirmed associations at 10 established HOMA-B/FG loci (Figure S5a, Table 1) ${ }^{13,21,43}$. The HOMA-IR GWIS provided three novel loci at LYPLAL1/SLC30A10, 
PER4, PPP1R3B and confirmed established loci at GCKR and IGF121 (Figure S5b, Table 1). Imputation using new tool SS-imp ${ }^{34}$ for summary statistics to $1000 \mathrm{G}$ density highlighted small difference from the lead variants at HOMA loci imputed to HapMap2 reference panel (Table S4). New lead 1000G-imputed variants at TCF7L2, ARAP1[STARD10] for HOMA-B, and at GCKR (rs11336847) for HOMA-IR, were in strong $\left(\mathrm{r}^{2}>0.8\right) \mathrm{LD}$, and at G6PC2 (rs580670 for HOMA-B in moderate $\left(\mathrm{r}^{2}>0.6\right)$ LD with HapMap2 lead variants (Table1).

GWIS requires FG/FI population mean estimates to infer summary statistics for HOMA-B/-IR, which vary across studies. We performed a set of sensitivity analyses, where we allowed mean estimates to fluctuate between reasonable values. These analyses indicated that within 1 standard deviation $(S D)$ from the two means $(M(S D)=$ $5.25(0.34) \mathrm{mmol} / \mathrm{l}$ for $\mathrm{FG}$ and $M(S D)=60.40(19.45) \mathrm{pmol} / \mathrm{l}$ for FI) 8 out of 11 HOMA-B loci were genome-wide significant in $100 \%$ of cases, except for GCK (72\%), SLC30A8 (86\%) and FOXA2 (55\%) with slight p-value drops to $4.7 \times 10^{-7}, 1.2 \times 10^{-7}$ and $2.7 \times 10^{-7}$ respectively; and all 5 HOMA-IR loci were genome-wide significant irrespective of FG/FI means variation (Figure S6).

Effects of established T2D, FG/FI and HbA1C loci on HOMA-B/-IR

We investigated the effects of established $102 \mathrm{~T} 2 \mathrm{D}, 50 \mathrm{FG} / \mathrm{FI}$ and $57 \mathrm{HbA} 1 \mathrm{C}^{44}$ loci on indices of $\beta$-cell function and insulin resistance (Figure 2, Tables S5-S7) ${ }^{20,45-48}$. As previous studies were performed using a number of reference panel imputations, the number of established T2D/FG/FI/HbA1c SNPs that map to HOMA-B/-IR GWIS results is smaller than the number of currently reported SNPs in the literature $\left(R^{2}>=0.9\right.$ for the proxy SNPs). The effects of T2D loci on HOMA indices followed a grouping previously defined by $\mathrm{us}^{29}$. The largest group of loci associated at least nominally with HOMA-B and 
primarily leading to reduced insulin secretion included 20 T2D loci. Some of these loci have at least a nominal effect on improved insulin sensitivity which likely reflects compensatory mechanisms observed in individuals without known T2D used for the HOMA analyses, as previously discussed ${ }^{29}$. The effects of T2D loci on HOMA index of insulin resistance (Figure $\mathbf{2 b}$ ) clearly demonstrate that, in addition to IRS1, GCKR, KLF14 and $P P A R G$, a number of more recently defined T2D loci, including PEPD, ANKRD55, BCL2, $A R L 15$ and GRB14 are related to this pathophysiological process. FTO, MC4R and NRXN3 are established body mass index (BMI) $l o c i^{49}$ and therefore their observed effects on fasting insulin resistance are likely to be driven through their primary effects on adiposity. Most of the established FG loci were at least nominally associated with the reduced $\beta$-cell function, as reflected by negative effect estimates for HOMA-B (Figure 2c), while FI loci were associated with decreased insulin sensitivity, i.e. provided positive effect estimates for HOMA-IR values (Figure 2d). Ten FG loci were genome-wide significant for HOMA-B (Figure 2c). FG level-increasing alleles at eight overlapping T2D loci (Figure 2a) represented by hyperglycaemia (MTNR1B, GCK), $\beta$-cell (SLC30A8, PROX1, ADCY5, GLIS3, TCF7L2), and proinsulin (ARAP1[STARD10]) loci, as well as the FG only loci that do not have established associations with T2D (G6PC2, FOXA2) were associated with reduced $\beta$-cell function through their primary effect on the set point of glucose. All FI loci were at least nominally associated with increased insulin resistance detected by HOMAIR (Figure 2d). At FI loci, we detected at least nominal effects on HOMA-B, which might be related to left truncation for FG values for these analyses which introduced seeming improvement in $\beta$-cell function ${ }^{29}$. The T2D effect allele in GCKR locus reflects solely the decreased insulin action, i.e. normal functioning of $\beta$-cells in the presence of increased insulin resistance (Figure $\mathbf{2 b})^{50,51}$. 
Nine of eleven genome-wide significant HOMA-B loci and four loci of suggestive significance (GCK, PDX1, CDKAL1, FTO) have established effects on HbA1c levels ${ }^{44}$ (Figure 2e). The HbA1c loci have been classified into erythrocytic or glycaemic, but a number of them remain unclassified. We suggest that five unclassified HbA1c loci $(P H B 2, F N D C 3 B$, GAS6, SYF2, CERS2) are glycaemic as they exert nominal effects on HOMA-B. Similarly, the abovementioned PHB2, FNDC3B, GAS6 as well as $A T A 2 B$ and FOXN2 are nominally associated with HOMA-IR and therefore are likely glycaemic.

\section{Enrichment in functional elements for HOMA-B/-IR associations}

The enrichment analysis using the GARFIELD software did not yield statistically significant co-localization signals neither for HOMA-B nor HOMA-IR. We observed suggestive evidence $(P<0.05)$ for an enrichment of the HOMA-B associated variants within several pathophysiologically relevant tissues, including blood, liver and brain. Both HOMA-B and HOMA-IR associated variants showed also suggestive evidence for enrichment in a number of annotations within K562 (human immortalised myelogenous leukemia cell line) and HepG2 (human immortalised liver carcinoma cell line) cells, including transcription start cites, weak enhancers among chromatin states, histone modifications, transcription factor binding cites and FAIRE-seq elements (Figure S7).

Genetic relationships between HOMA indices and other phenotypes.

LDSC $^{33}$ was applied to estimate the genome-wide genetic correlation between HOMA-B/IR and 36 other phenotypes falling into five broad domains (Table S8), including, T2D, 13 glycaemic traits, four blood lipids, four obesity traits, nine phenotypes indicative of T2D complications and five inflammation markers (Figure 3). HOMA-B showed positive and strong $\left(\mathrm{r}_{\mathrm{g}}=0.68[\mathrm{SE}=0.07], \mathrm{P}=4.33 \times 10^{-21}\right)$ genetic correlation with HOMA-IR in our 
data. We identified the strongest novel genetic correlation (FDR-adjusted to correct for multiple testing at $\mathrm{P}<0.05$ ) in the domain of inflammatory markers, specifically between HOMA-IR and PAI-1 $\left(\mathrm{r}_{\mathrm{g}}=0.92, \mathrm{P}=2.13 \times 10^{-4}\right)$, usually observed at higher levels in people with obesity and metabolic syndrome ${ }^{39}$. Similarly, HOMA-B directly correlated with PAI$1\left(\mathrm{r}_{\mathrm{g}}=0.78, \mathrm{P}=2.54 \times 10^{-3}\right)$. CRP positively correlated with both HOMA indices ( $\mathrm{r}_{\mathrm{gHOMA}-\mathrm{B} /-}$ IR $=0.28 / 0.33$, Рнома-в/-IR $=3.65 \times 10^{-3} / 4.67 \times 10^{-3}$ ), while adiponectin showed significant inverse correlation with HOMA-IR only $\left(\mathrm{r}_{\mathrm{g}}=-0.30, \mathrm{P}=0.012\right)$.

For HOMA-IR, we saw significant correlations with FI $\left(\mathrm{r}_{\mathrm{g}}=0.98, \mathrm{P}=<0.001\right)$, FG $\left(r_{g}=0.49, P=1.37 \times 10^{-9}\right), H_{b A} c\left(r_{g}=0.28, P=0.02\right)$ and $T 2 D\left(r_{g}=0.56, P=2.31 \times 10^{-9}\right)$, in accordance with epidemiological observations. The genetic correlation between HOMAB from our largest to date GWAS confirmed initial observations ${ }^{18}$ on the strong genetic correlation with FI $\left(\mathrm{r}_{\mathrm{g}}=0.80, \mathrm{P}=8.77 \times 10^{-72}\right)$. In accordance with previous reports, the correlation with $\mathrm{FG}\left(\mathrm{rg}_{\mathrm{g}}=-0.32, \mathrm{P}=0.05\right)$ and relationship with $\mathrm{T} 2 \mathrm{D}\left(\mathrm{r}_{\mathrm{g}}=0.05, \mathrm{P}=0.71\right)$ were not significant after multiple testing corrections ${ }^{18,52}$. Other indices of glucose homeostasis, including the insulin sensitivity index, ISI, without adjustment for BMI, were

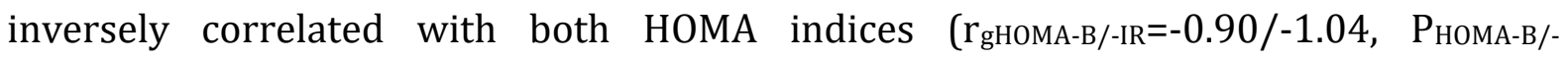
$\mathrm{IR}=1.20 \times 10^{-5} / 2.71 \times 10^{-8}$ ). We did not find significant genetic correlations between HOMAB/-IR and other glycaemic traits, which is likely due to small GWAS sample sizes for those phenotypes (Table S8). Triglycerides (TG) and all obesity traits were directly correlated with both HOMA-B/-IR, similarly to a previous report that used enrichment analysis approaches $^{13}$. We found no significant genetic correlations between HOMA-B/-IR and traits in the domain of T2D complications with the exception of Estimated Glomerular

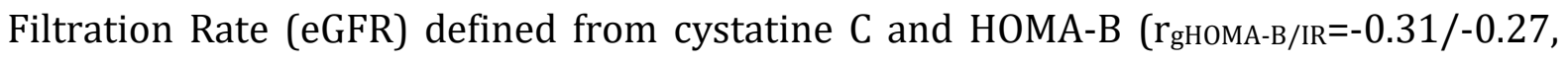
PномA-B/IR=0.03/0.05). 


\section{Effects of fasting glycaemic trait loci on PAI-1 variability}

We followed up the observation from genome-wide genetic correlation estimates by evaluation of the effects of FG/FI loci on PAI-1 and HOMA-B and HOMA-IR, respectively (Figure 4). While only four FG loci (PPP1R2B, MTNR1B, CRY2 and GCKR) showed at least nominally significant effects on PAI-1 ${ }^{\text {ref39, }}$, the direction of their effects was variable. Interestingly, among FI loci, at least half of the loci had nominal association with PAI-1. At only two loci (PELO and GCKR), FI increasing allele was associated with lower PAI-1 levels, GCKR variant effect being in line with its established mutational mechanism leading at the same time to higher FG and lower triglycerides levels ${ }^{51}$. At the same time, among four established PAI-1 loci, rs1801282-A (coding SNP, Pro12Ala) at PPARG is an established T2D risk ${ }^{53}$ and insulin resistance ${ }^{29}$ variant leading to higher PAI-1 levels.

\section{Discussion}

We report the largest GWAS to date of HOMA-B and HOMA-IR indices in nondiabetic individuals of European origin, inferred analytically from FG and FI GWAS metaanalysis summary statistics and imputed using novel methodology ${ }^{34}$ to 1000 Genomes Project variant density. The application of GWIS methodology brought the total count of HOMA-B/-IR loci to $11 / 5$, respectively, and brought the analytical sample size up to $\sim 75,240$ individuals. This GWIS-based analysis revealed one novel HOMA-B and three novel HOMA-IR loci. We highlighted the strongest novel positive genetic correlations between non-glycaemic trait PAI-1 and HOMA-B/-IR. We also reported novel positive genetic relationships between CRP and HOMA-B/-IR, as well as inverse relationships between adiponectin and HOMA-IR. We demonstrated that GWIS of both HOMAs produced more precise SNP effect parameter estimates, and thus gained power, compared to the previous GWAS ${ }^{21}$. The results of LDSC $^{33}$ analysis confirmed that the gain 
in power in GWIS did not arise from the introduction of spurious inflation due to population stratification or other biases.

Novel HOMA-B/-IR loci, including FOXA2, LYPLAL1/SLC30A10, PPP1R3B, are established for FG or FI, while HOMA-IR locus at PER4 encoding for Period Circadian Clock 3 Pseudogene is novel. The lead variant rs10224545 at PER4 also showed a genome-wide significant association with FI in our data. This region was not covered on Illumina Metabochip and therefore was not detected by a larger study from the MAGIC investigators ${ }^{13}$. Similarly to $M T N R 1 B^{23,54}$ and $C R Y 2^{21}$ loci, both affecting FG levels and related to indices of $\beta$-cell function, the association with insulin resistance at PER4 gene provides an additional link between circadian rhythmicity and energy metabolism ${ }^{55}$. Overall, nine HOMA-B loci at ADCY5, DGKB, GCK, SLC30A8, GLIS3, TCF7L2, ARAP1, LYPLAL1/SLC30A10 and MTNR1B and one HOMA-IR locus at GCKR are also established as contributing to the risk of $\mathrm{T}_{2} \mathrm{D}^{47}$. Among the remaining, two lead variants (rs174555, rs4240624 at $F A D S 1 / 2 / 3$ and $P P P 1 R 3 B$, respectively) are only nominally $(\mathrm{P}<0.05)$ associated with T2D risk ${ }^{47}$. Other HOMA-B loci, such as G6PC2, are established for glycated haemoglobin ( $\mathrm{HbA1}$ ) levels, in addition to $\mathrm{FG}$, or for a wide range of glycaemic, lipid, metabolomic traits, such as variants within FADS1 refs21,56,57. Among novel HOMA-IR loci, LYPLAL1/SLC30A10 is an established FI (rs2820436, $\mathrm{R}^{2}=1$ ) ref43 $^{\text {and obesity locus }}$ known for its effects on waist-to hip ratio (WHR) in women (rs2820443, $\left.\mathrm{R}^{2}=0.44\right)^{\text {ref58, }}$ while associations at $P P P 1 R 3 B$ are established for several glycaemic traits, including FI (rs983309, r2=0.75; rs2126259, r2=0.85), FG (rs983309, r2=0.75) and 2-hour postprandial glucose, $2 \mathrm{hrGlu}(\mathrm{rs} 11782386, \mathrm{r} 2=0.60)$.

High insulin and glucose levels, insulin resistance and $\beta$-cell dysfunction characterize the pathophysiology of T2D. However, the exact mechanism of genetic interrelationships between the metabolic processes and disease is still unclear. Amongst 
the established T2D loci $45,46,48$ we investigated in relation to HOMA indices GWIS results (Tables S5-S7), only 41/30 have at least nominal effect on HOMA-B/-IR variability, respectively, highlighting an incomplete overlap between genetic variants affecting normal glucose homeostasis and processes involved in T2D pathogenesis. Insulin resistance and $\beta$-cell function may have distinct impact on susceptibility to $\mathrm{T}^{2} \mathrm{D}^{28}$, and mechanistically T2D loci can be related to a specific biological process affecting insulin secretion, resistance or processing ${ }^{59}$. Effects of T2D loci follow the main subdivisions into $\beta$-cell, hyperglycaemia, proinsulin and insulin resistance groups ${ }^{29}$. The group of established T2D loci that influence insulin resistance or fasting insulin has been undercharacterised, most probably, due to low power of respective endophenotype GWAS meta-analyses ${ }^{21,60}$. Empowered by analytically inferred HOMA-IR GWAS, we demonstrated that the group on insulin resistance loci is larger than described before and comparable to that of $\beta$-cell loci. As expected from epidemiological studies, FG loci also affect HOMA-B values (Figure S5), whereas FI variants have most prominent effect on HOMA-IR.

We confirmed the evidence of shared genetic effects between HOMA-B and T2D risk at several loci, manifested by inverse relationships ${ }^{28,29}$. However, the effect of left truncation for FG levels to define non-diabetic individuals in general population leads to seemingly improved $\beta$-cell function through positive HOMA-B values for the insulin resistance loci. In fact, we did not observe any genetic correlation between HOMA-B and T2D using summary statistics from the latest GWAS meta-analyses. In parallel, we however demonstrated that HOMA-B loci, associated with a decrease in $\beta$-cell function, exert their effects by both increasing and decreasing insulin resistance (Figure 2a-b).

Inflammation markers PAI-1 and CRP showed strong positive genetic correlation with both HOMA-B/-IR measures. The correlation of these markers was stronger with 
fasting insulin resistance. CRP is a marker of increased cardiovascular risk ${ }^{61,62}$ and PAI-1 is associated with $\mathrm{CAD}^{63}$ and myocardial infarction ${ }^{64}$, both high risk factors in T2D. Importantly, PAI-1 is a fibrinolytic and inflammatory marker, which was proposed and confirmed in epidemiological studies as an independent risk factor for T2D ${ }^{65,66}$. In our study among non-glycaemic traits, PAI-1 showed the strongest genetic correlation with fasting insulin resistance, a pathophysiological process leading to T2D. Moreover, when we looked at the individual effects of FG/FI variants on PAI-1 variability, variant alleles associated with higher FI grouped around increased levels of PAI-1, while there was no clear pattern of such an effect for FG variants. From pathophysiological point of view, the clustering of hyperinsulinaemia, dysglycemia, dyslipidemia, and hypertension as cardiovascular risk endophenotypes in T2D occurs around insulin resistance, and in the presence of elevated PAI-1 levels, leads to fibrinolytic dysfunction, increased thrombotic risk $^{17,67}$, and induces local or systemic low-grade inflammation. Taken together, evidence from our study provides novel genetic support for the need to dissect in better detail these deficient mechanisms in the early pathogenesis of T2D.

Noteworthy, we observed no significant genetic correlation between HOMA-B/-IR and CAD, hypertension, SBP and DBP, which could indicate that the associations between HOMA-B/-IR and PAI-1 and CRP are strictly due to inflammatory pathways involved in T2D risk. Contrary to expectations from epidemiological studies, we neither observed correlation between adiponectin and HOMA-B for genetic effects using the GWAS summary statistics, whilst the respective genetic correlation with HOMA-IR may be through its association with obesity, since adiponectin is secreted in adipose tissue. Alternatively, it can be due to its involvement in inflammatory processes in T2D pathogenesis $^{68}$. Analysis of CKD and its marker eGFR based on creatinine did not yield statistically significant genetic correlations; only eGFR based on cystatine C for HOMA-B 
did so. The latter could reflect the association between cystatine and metabolic syndrome ${ }^{69}$, whose definition includes T2D risk factors such as insulin resistance and abnormal fat distribution ${ }^{70}$.

Finally, we reported novel significant positive genetic correlation between HOMAIR and T2D, and our estimates of genetic correlations are concordant with established genome-wide relationships between HOMA-B and FG/FI/BMI/T2D and HOMA-IR and FG/FI/BMI ${ }^{18}$. The positive genetic correlations for $\mathrm{TG}$ and negative for high density lipoprotein cholesterol (HDL-C) with HOMA-B/-IR reflect the conditions of diabetic dyslipidaemia, characterised by increased levels of TG and decreased levels of HDL cholesterol $^{71}$. The role of obesity and adiposity 43,72 , in particular, in the risk of T2D is well established and was reflected in significant genetic correlations between HOMA-B/-IR and obesity related traits, such as BMI, WHR, waist circumference (WC), hip circumference (HIP), in our study. In addition, adiposity loci FTO, MC4R and NRXN3 were at least nominally associated with HOMA-B/-IR in our analysis not adjusted for BMI.

Our study provides additional evidence for the GWIS method as a powerful tool for future GWAS studies of analytically derived phenotypes. Our work also suggests that GWAS meta-analysis of summary statistics is a useful source of information for follow-up analyses, including inferences about genetic correlations and mechanistic characterisation of the specific trait pathophysiology through their effects on other related phenotypes. 
bioRxiv preprint doi: https://doi.org/10.1101/496802; this version posted December 28,2018 . The copyright holder for this preprint (which was not certified by peer review) is the author/funder, who has granted bioRxiv a license to display the preprint in perpetuity. It is made available under aCC-BY-NC-ND 4.0 International license.

\section{Data Access:}

The GWAS summary statistics for the MAGIC investigators study of FG and FI and the present study results will be deposited on the consortium web site www.magicinvestigators.org. 
Acknowledgments: IOF is supported by Biobanking and Biomolecular Resources Research Infrastructure (BBMRI-NL, NWO 184.021.007). I.P. is funded by the World Cancer Research Fund (WCRF UK) and World Cancer Research Fund International (2017/1641), the Wellcome Trust (WT205915), and the European Union's Horizon 2020 research and innovation programme (DYNAhealth, project number 633595). MGN is supported by Royal Netherlands Academy of Science Professor Award (PAH/6635) granted to D.I. Boomsma. Mike A. Nalls' participation is supported by a consulting contract between Data Tecnica International and the National Institute on Aging, NIH, Bethesda, MD, USA. Data on glycaemic traits have been contributed by MAGIC investigators and have been downloaded from www.magicinvestigators.org.

Author contributions: Conceived and designed the experiments: I.P. and D.I.B.; Analysed the data: Meta-analyses: R.M., D.I.C., S.G., J.H., M.A.N., C.J.O., G.P., P.M.R., MAGIC Investigators and CHARGE Inflammation working group; analytical derivation of HOMAB/HOMA-IR GWIS summary statistics: I.O.F, M.G.N; central analysis group: I.O.F, L.Z, M.K., M.G.N, I.P., and XC-Pleiotropy Group. Wrote the first draft of the manuscript: I.O.F., J.J.H., M.G.N, I.P. and D.I.B. Contributed to the writing of the manuscript: I.O.F., M.G.N., J.J.H., L.Z, Z.B., D.I.C., S.G., J.H., M.A.N., C.J.O., G.P., P.M.R., M.K., R.M., I.P., D.I.B..

Conflict of interest: Dr. Nalls consults for Illumina Inc, the Michael J. Fox Foundation, University of California Healthcare and Genoom Health among others. Other authors declare no conflict of interest. 
Table 1. Comparison of HOMA-B/-IR and FG/FI effects across genome-wide significant loci

\begin{tabular}{|c|c|c|c|c|c|c|c|}
\hline SNP & CHR & Locus name & $\begin{array}{l}\text { Alleles } \\
\text { (effect } \\
\text { other) }\end{array}$ & Effect (SE), p-value & $\mathrm{N}$ & Effect (SE), p-value & $\mathrm{N}$ \\
\hline & & & & HOMA-B & & FG & \\
\hline rs560887 & 2 & G6PC2 & $\mathrm{C} / \mathrm{T}$ & $-0.046(0.0033), 4.74 \times 10^{-46}$ & 75,173 & $0.069(0.0027), 2.41 \times 10^{-144}$ & 88,202 \\
\hline rs11708067 & 3 & $A D C Y 5$ & $\mathrm{~A} / \mathrm{G}$ & $-0.024(0.0038), 2.50 \times 10^{-10}$ & 75,227 & $0.022(0.0031), 5.55 \times 10^{-13}$ & 88,301 \\
\hline rs10258074 & 7 & $D G K B$ & $\mathrm{~A} / \mathrm{T}$ & $0.019(0.0031), 2.55 \times 10^{-10}$ & 75,202 & $-0.028(0.0025), 1.18 \times 10^{-27}$ & 88,240 \\
\hline rs3757840 & 7 & $G C K$ & $\mathrm{G} / \mathrm{T}$ & $0.02(0.0033), 1.98 \times 10^{-09}$ & 64,352 & $-0.037(0.0029), 3.66 \times 10^{-38}$ & 72,887 \\
\hline rs3802177 & 8 & SLC30A8 & $\mathrm{G} / \mathrm{A}$ & $-0.021(0.0036), 2.55 \times 10^{-09}$ & 68,044 & $0.029(0.0030), 1.24 \times 10^{-21}$ & 76,819 \\
\hline rs7034200 & 9 & GLIS3 & $\mathrm{A} / \mathrm{C}$ & $-0.019(0.003), 2.96 \times 10^{-10}$ & 74,669 & $0.016(0.0025), 2.32 \times 10^{-10}$ & 87,749 \\
\hline
\end{tabular}




\begin{tabular}{|c|c|c|c|c|c|c|c|}
\hline rs7903146 & 10 & TCF7L2 & $\mathrm{C} / \mathrm{T}$ & $0.03(0.0034), 7.41 \times 10^{-19}$ & 75,212 & $-0.022(0.0028), 1.04 \times 10^{-14}$ & 88,262 \\
\hline rs10830963 & 11 & MTNR1B & $\mathrm{C} / \mathrm{G}$ & $0.039(0.0038), 9.34 \times 10^{-25}$ & 68,612 & $-0.073(0.0032), 2.37 \times 10^{-113}$ & 77,412 \\
\hline rs1552224 & 11 & ARAP1[STARD10] & $\mathrm{C} / \mathrm{A}$ & $0.025(0.004), 4.28 \times 10^{-10}$ & 75,207 & $-0.023(0.0034), 5.56 \times 10^{-12}$ & 88,250 \\
\hline rs174555 & 11 & $F A D S 1 / 2 / 3$ & $\mathrm{~T} / \mathrm{C}$ & $-0.021(0.0033), 8.97 \times 10^{-11}$ & 75,237 & $0.021(0.0027), 2.17 \times 10^{-14}$ & 88,322 \\
\hline \multirow[t]{2}{*}{ rs5029909 } & 20 & FOXA2 & $\mathrm{C} / \mathrm{T}$ & $-0.044(0.0079), 2.70 \times 10^{-08}$ & 68,584 & $0.044(0.0068), 1.49 \times 10^{-10}$ & 79,095 \\
\hline & & & & \multicolumn{2}{|l|}{ HOMA-IR } & FI & \\
\hline rs2605101 & 1 & LYPLAL1/SLC30A10 & $\mathrm{A} / \mathrm{T}$ & $0.019(0.0034), 3.78 \times 10^{-08}$ & 75,238 & $0.017(0.0032), 1.46 \times 10^{-07}$ & 64,091 \\
\hline rs780093 & 2 & GCKR & $\mathrm{C} / \mathrm{T}$ & $0.024(0.0032), 1.06 \times 10^{-13}$ & 75,226 & $0.018(0.0030), 1.73 \times 10^{-09}$ & 64,091 \\
\hline rs10224545 & 7 & PER4 & $\mathrm{T} / \mathrm{C}$ & $-0.031(0.0056), 2.60 \times 10^{-08}$ & 71,951 & $-0.029(0.0052), 2.92 \times 10^{-08}$ & 64,091 \\
\hline rs4240624 & 8 & $P P P 1 R 3 B$ & $\mathrm{~A} / \mathrm{G}$ & $-0.035(0.0055), 2.73 \times 10^{-10}$ & 68,617 & $-0.030(0.0051), 4.25 \times 10^{-09}$ & 60,824 \\
\hline rs2114912 & 12 & $I G F 1$ & $\mathrm{~T} / \mathrm{G}$ & $0.026(0.0044), 3.08 \times 10^{-09}$ & 74,634 & $0.024(0.0041), 3.76 \times 10^{-09}$ & 63,539 \\
\hline
\end{tabular}




\section{References:}

1. IDF. (International Diabetes Federation), Diabetes Atlas, 8th edition. 2017.

2. Stumvoll M, Goldstein BJ, van Haeften TW. Pathogenesis of type 2 diabetes. Endocrine research. 2007;32(1-2):19-37.

3. Matthews D, Hosker J, Rudenski A, Naylor B, Treacher D, Turner R. Homeostasis model assessment: insulin resistance and $\beta$-cell function from fasting plasma glucose and insulin concentrations in man. Diabetologia. 1985;28(7):412-419.

4. Tang Q, Li X, Song P, Xu L. Optimal cut-off values for the homeostasis model assessment of insulin resistance (HOMA-IR) and pre-diabetes screening: Developments in research and prospects for the future. Drug discoveries \& therapeutics. 2015;9(6):380-385.

5. Morimoto A, Tatsumi Y, Soyano F, et al. Increase in homeostasis model assessment of insulin resistance (HOMA-IR) had a strong impact on the development of type 2 diabetes in Japanese individuals with impaired insulin secretion: the saku study. PloS one. 2014;9(8):e105827.

6. Haffner SM, Gonzalez C, Miettinen H, Kennedy E, Stern MP. A prospective analysis of the HOMA model: the Mexico City Diabetes Study. Diabetes care. 1996;19(10):1138-1141.

7. Kasuga M. Insulin resistance and pancreatic $\beta$ cell failure. The Journal of clinical investigation. 2006;116(7):1756-1760.

8. Kahn S. The relative contributions of insulin resistance and beta-cell dysfunction to the pathophysiology of type 2 diabetes. Diabetologia. 2003;46(1):3-19.

9. Sladek R, Prokopenko I. Genome-wide association studies of type 2 diabetes. The Genetics of Type 2 Diabetes and Related Traits: Springer; 2016:13-61.

10. Song Y, Manson JE, Tinker L, et al. Insulin sensitivity and insulin secretion determined by homeostasis model assessment and risk of diabetes in a multiethnic cohort of women: the Women's Health Initiative Observational Study. Diabetes care. 2007;30(7):1747-1752.

11. Cerf ME. Beta cell dysfunction and insulin resistance. Frontiers in endocrinology. 2013;4:37.

12. Fall $T$, Hägg $S$, Mägi $R$, et al. The role of adiposity in cardiometabolic traits: a Mendelian randomization analysis. PLoS Med. 2013;10(6):e1001474.

13. Scott RA, Lagou $V$, Welch RP, et al. Large-scale association analyses identify new loci influencing glycemic traits and provide insight into the underlying biological pathways. Nature genetics. 2012;44(9):991-1005.

14. Kraja AT, Chasman DI, North KE, et al. Pleiotropic genes for metabolic syndrome and inflammation. Molecular genetics and metabolism. 2014;112(4):317-338.

15. Kolb H, Mandrup-Poulsen T. The global diabetes epidemic as a consequence of lifestyle-induced low-grade inflammation. Diabetologia. 2010;53(1):10-20.

16. Donath MY, Shoelson SE. Type 2 diabetes as an inflammatory disease. Nature Reviews Immunology. 2011;11(2):98-107.

17. Cesari M, Pahor M, Incalzi RA. Plasminogen activator inhibitor-1 (PAI-1): a key factor linking fibrinolysis and age-related subclinical and clinical conditions. Cardiovascular therapeutics. 2010;28(5):e72-e91. 
18. Bulik-Sullivan B, Finucane $\mathrm{HK}$, Anttila V, et al. An atlas of genetic correlations across human diseases and traits. Nature genetics. 2015.

19. Vattikuti S, Guo J, Chow CC. Heritability and genetic correlations explained by common SNPs for metabolic syndrome traits. PLoS Genet. 2012;8(3):e1002637.

20. Marullo L, Moustafa JSE-S, Prokopenko I. Insights into the genetic susceptibility to type 2 diabetes from genome-wide association studies of glycaemic traits. Current diabetes reports. 2014;14(11):1-17.

21. Dupuis J, Langenberg C, Prokopenko I, et al. New genetic loci implicated in fasting glucose homeostasis and their impact on type 2 diabetes risk. Nature genetics. 2010;42(2):105-116.

22. Bouatia-Naji N, Rocheleau G, Van Lommel L, et al. A polymorphism within the G6PC2 gene is associated with fasting plasma glucose levels. Science. 2008;320(5879):1085-1088.

23. Prokopenko I, Langenberg C, Florez JC, et al. Variants in MTNR1B influence fasting glucose levels. Nature genetics. 2009;41(1):77-81.

24. Chen G, Bentley A, Adeyemo A, et al. Genome-wide association study identifies novel loci association with fasting insulin and insulin resistance in African Americans. Human molecular genetics. 2012:dds282.

25. Strawbridge RJ, Dupuis J, Prokopenko I, et al. Genome-wide association identifies nine common variants associated with fasting proinsulin levels and provides new insights into the pathophysiology of type 2 diabetes. Diabetes. 2011;60(10):2624-2634.

26. Prokopenko I, Poon W, Mägi R, et al. A central role for GRB10 in regulation of islet function in man. PLoS Genet. 2014;10(4):e1004235.

27. Walford GA, Gustafsson S, Rybin D, et al. Genome-wide association study of the modified Stumvoll Insulin Sensitivity Index identifies BCL2 and FAM19A2 as novel insulin sensitivity loci. Diabetes. 2016:db160199.

28. Voight BF, Scott LJ, Steinthorsdottir V, et al. Twelve type 2 diabetes susceptibility loci identified through large-scale association analysis. Nature genetics. 2010;42(7):579-589.

29. Dimas AS, Lagou V, Barker A, et al. Impact of type 2 diabetes susceptibility variants on quantitative glycemic traits reveals mechanistic heterogeneity. Diabetes. 2014:DB_130949.

30. Nieuwboer HA, Pool R, Dolan CV, Boomsma DI, Nivard MG. GWIS: GenomeWide Inferred Statistics for Functions of Multiple Phenotypes. The American Journal of Human Genetics. 2016.

31. Wallace TM, Levy JC, Matthews DR. Use and abuse of HOMA modeling. Diabetes care. 2004;27(6):1487-1495.

32. Pasaniuc B, Price AL. Dissecting the genetics of complex traits using summary association statistics. Nature reviews genetics. 2016.

33. Bulik-Sullivan BK, Loh P-R, Finucane HK, et al. LD Score regression distinguishes confounding from polygenicity in genome-wide association studies. Nature genetics. 2015;47(3):291-295.

34. Rüeger S, McDaid A, Kutalik Z. Improved imputation of summary statistics for realistic settings. bioRxiv. 2018:203927.

35. Iotchkova V, Huang J, Morris JA, et al. Discovery and refinement of genetic loci associated with cardiometabolic risk using dense imputation maps. Nat Genet. Nov 2016;48(11):1303-1312. 
36. Pattaro C, Teumer A, Gorski M, et al. Genetic associations at 53 loci highlight cell types and biological pathways relevant for kidney function. Nature communications. 2016;7.

37. Newton-Cheh C, Johnson T, Gateva V, et al. Genome-wide association study identifies eight loci associated with blood pressure. Nature genetics. 2009;41(6):666-676.

38. Dastani Z, Hivert M-F, Timpson N, et al. Novel loci for adiponectin levels and their influence on type 2 diabetes and metabolic traits: a multi-ethnic metaanalysis of 45,891 individuals. PLoS Genet. 2012;8(3):e1002607.

39. Huang J, Sabater-Lleal M, Asselbergs FW, et al. Genome-wide association study for circulating levels of plasminogen activator inhibitor-1 (PAI-1) provides novel insights into the regulation of PAI-1. Blood. 2012:blood-2012-2006436188.

40. Dehghan A, Dupuis J, Barbalic M, et al. Meta-Analysis of Genome-Wide Association Studies in> 80000 Subjects Identifies Multiple Loci for C-Reactive Protein LevelsClinical Perspective. Circulation. 2011;123(7):731-738.

41. Paré G, Ridker PM, Rose $\mathrm{L}$, et al. Genome-wide association analysis of soluble ICAM-1 concentration reveals novel associations at the NFKBIK, PNPLA3, RELA, and SH2B3 loci. PLoS Genet. 2011;7(4):e1001374.

42. Nalls MA, Couper DJ, Tanaka T, et al. Multiple loci are associated with white blood cell phenotypes. PLoS Genet. 2011;7(6):e1002113.

43. Manning AK, Hivert M-F, Scott RA, et al. A genome-wide approach accounting for body mass index identifies genetic variants influencing fasting glycemic traits and insulin resistance. Nature genetics. 2012;44(6):659-669.

44. Wheeler E, Leong A, Liu C-T, et al. Impact of common genetic determinants of Hemoglobin A1c on type 2 diabetes risk and diagnosis in ancestrally diverse populations: A transethnic genome-wide meta-analysis. PLoS medicine. 2017;14(9):e1002383.

45. Florez JC. The Genetics of Type 2 Diabetes and Related Traits.

46. Liu C-T, Raghavan S, Maruthur $\mathrm{N}$, et al. Trans-ethnic meta-analysis and functional annotation illuminates the genetic architecture of fasting glucose and insulin. The American Journal of Human Genetics. 2016;99(1):56-75.

47. Scott RA, Scott LJ, Mägi R, et al. An Expanded Genome-Wide Association Study of Type 2 Diabetes in Europeans. Diabetes. 2017:db161253.

48. Zhao $W$, Rasheed A, Tikkanen E, et al. Identification of new susceptibility loci for type 2 diabetes and shared etiological pathways with coronary heart disease. Nat Genet. 2017; advance online publication.

49. Locke AE, Kahali B, Berndt SI, et al. Genetic studies of body mass index yield new insights for obesity biology. Nature. 2015;518(7538):197.

50. Raimondo A, Rees MG, Gloyn AL. Glucokinase regulatory protein: complexity at the crossroads of triglyceride and glucose metabolism. Current opinion in lipidology. 2015;26(2):88.

51. Beer NL, Tribble ND, McCulloch LJ, et al. The P446L variant in GCKR associated with fasting plasma glucose and triglyceride levels exerts its effect through increased glucokinase activity in liver. Human molecular genetics. 2009;18(21):4081-4088.

52. Benjamini Y, Hochberg Y. Controlling the false discovery rate: a practical and powerful approach to multiple testing. Journal of the royal statistical society. Series B (Methodological). 1995:289-300. 
53. Altshuler D, Hirschhorn JN, Klannemark M, et al. The common PPAR $\gamma$ Pro12Ala polymorphism is associated with decreased risk of type 2 diabetes. Nature genetics. 2000;26(1):76.

54. Bouatia-Naji N, Bonnefond A, Cavalcanti-Proença C, et al. A variant near MTNR1B is associated with increased fasting plasma glucose levels and type 2 diabetes risk. Nature genetics. 2009;41(1):89.

55. Renström F, Koivula RW, Varga TV, et al. Season-dependent associations of circadian rhythm-regulating loci (CRY1, CRY2 and MTNR1B) and glucose homeostasis: the GLACIER Study. Diabetologia. 2015;58(5):997-1005.

56. Kettunen J, Tukiainen $T$, Sarin A-P, et al. Genome-wide association study identifies multiple loci influencing human serum metabolite levels. Nature genetics. 2012;44(3):269-276.

57. Teslovich TM, Musunuru K, Smith AV, et al. Biological, clinical and population relevance of 95 loci for blood lipids. Nature. 2010;466(7307):707-713.

58. Shungin D, Winkler TW, Croteau-Chonka DC, et al. New genetic loci link adipose and insulin biology to body fat distribution. Nature. 2015;518(7538):187-196.

59. Dimas AS, Lagou V, Barker A, et al. Impact of type 2 diabetes susceptibility variants on quantitative glycemic traits reveals mechanistic heterogeneity. Diabetes. 2013:DB_130949.

60. Florez J. Newly identified loci highlight beta cell dysfunction as a key cause of type 2 diabetes: where are the insulin resistance genes? Diabetologia. 2008;51(7):1100-1110.

61. Wang J, Tan G-J, Han L-N, Bai Y-Y, He M, Liu H-B. Novel biomarkers for cardiovascular risk prediction. Journal of Geriatric Cardiology: JGC. 2017;14(2):135.

62. Lacey B, Herrington WG, Preiss D, Lewington S, Armitage J. The Role of Emerging Risk Factors in Cardiovascular Outcomes. Current Atherosclerosis Reports. 2017;19(6):28.

63. Song C, Burgess S, Eicher JD, et al. Causal effect of plasminogen activator inhibitor type 1 on coronary heart disease. Journal of the American Heart Association. 2017;6(6):e004918.

64. Kohler HP, Grant PJ. Plasminogen-activator inhibitor type 1 and coronary artery disease. New England Journal of Medicine. 2000;342(24):1792-1801.

65. Yarmolinsky J, Barbieri NB, Weinmann T, Ziegelmann PK, Duncan BB, Schmidt MI. Plasminogen activator inhibitor- 1 and type 2 diabetes: a systematic review and meta-analysis of observational studies. Scientific reports. 2016;6:17714.

66. Festa A, D'Agostino R, Tracy RP, Haffner SM. Elevated levels of acute-phase proteins and plasminogen activator inhibitor-1 predict the development of type 2 diabetes: the insulin resistance atherosclerosis study. Diabetes. 2002;51(4):1131-1137.

67. Grant P. Diabetes mellitus as a prothrombotic condition. Journal of internal medicine. 2007;262(2):157-172.

68. Fantuzzi G. Adiponectin and inflammation: consensus and controversy. Journal of Allergy and Clinical Immunology. 2008;121(2):326-330.

69. Magnusson M, Hedblad B, Engström G, Persson M, Nilsson P, Melander O. High levels of cystatin $C$ predict the metabolic syndrome: the prospective Malmö Diet and Cancer Study. Journal of internal medicine. 2013;274(2):192-199. 
70. Alberti KGMM, Zimmet $P$, Shaw J. Metabolic syndrome-a new world-wide definition. A consensus statement from the international diabetes federation. Diabetic medicine. 2006;23(5):469-480.

71. Bitzur R, Cohen H, Kamari Y, Shaish A, Harats D. Triglycerides and HDL Cholesterol Stars or second leads in diabetes? Diabetes care. 2009;32(suppl 2):S373-S377.

72. Kahn SE, Hull RL, Utzschneider KM. Mechanisms linking obesity to insulin resistance and type 2 diabetes. Nature. 2006;444(7121):840-846. 


\section{Figure Titles and Legends.}

Figure 1. Comparison between GWAS meta-analysis of primary phenotypes and GWIS of derived phenotypes. Input phenotypes are fasting glucose (FG) and fasting insulin (FI), output phenotypes are Homeostasis Model Assessment of $\beta$-cell function (HOMA-B) and insulin resistance (HOMA-IR). a) Examples of how FG and FI measures could partially overlap within one cohort (e.g. Study A), whereas some cohorts could only have measures of FG (Study C) or FI (Study B). Study A could participate in future GWAS by computing HOMA-B/IR for overlapping individuals, although the sample size will be decreased in comparison to original FG and FI phenotypes. Study B and C cannot contribute to HOMA-B/IR GWAS, thus reducing the sample size of GWAS meta-analysis of HOMA-B/IR to a larger extent. b) Formulae to compute HOMA-B/IR from FG and FI measures. c) Conventional GWAS approach, where HOMA-B/IR would be computed based on individual cohort studies, undergoing the reduction of the sample size, if FG and FI are not measured in the same individual. GWAS summary statistics would be then computed on reduced sample size. d) GWIS approach, where FG and FI meta-analyses summary statistics are computed separately with maximum sample size available. HOMA-B/-IR summary statistics are then calculated from FG and FI meta-analyses results. The sample size of derived phenotypes are computed as geometric mean between FG and FI sample sizes. 


\section{Figure 2. Effects of established T2D (a,b), FG/FI (c,d) and HbA1c (e,f) loci on}

HOMA-B/-IR. Legend: X-axis and Y-axes represent the direction of loci effects on $\beta$ cell function and insulin resistance as reflected by HOMA-B/-IR, respectively. Note, DUSP9 T2D locus is not included on the Figure $2(\mathrm{a}, \mathrm{b})$ as it resides on $\mathrm{X}$ chromosome. Effects of HOMA-B/-IR SNPs correspond to the risk alleles of either T2D, FG/FI or HbA1c SNPs. The largest group of 20 T2D loci $(D G K B, C D K N 2 A / B, C 2 C D 4 A, T P 53 I N P 1$, HHEX/IDE, UBE2E2, ANK1, SPRY2, TMEM163, TMEM154, HNF4A, PROX1, THADA, $B R A F, M N X 1, H N F 1 B(T C F 2), G P S M 1, I G F 2 B P 2, K C N Q 1, K L)$ is associated at least nominally with HOMA-B and is primarily leading to reduced insulin secretion. The strongest effects on HOMA-B were observed for the loci characterised by the reduced $\beta$-cell function (SLC30A8, ADCY5, TCF7L2, GLIS3 and CDKAL1), reduced proinsulin production and insulin secretion at ARAP1(STARD10), and those related to fasting hyperglycaemia (MTNR1B and $G C K$ ). 
Figure 3. Genetic correlations ( $r_{g}$ ) between HOMA-B/-IR and 13 glycaemic, 17 cardiometabolic traits, five inflammation markers, and T2D. Genetic correlations reaching statistical significance (FDR-adjusted $\mathrm{P}<0.05$ ) are indicated in bold black in contrast to non-significant correlations reported in italic grey. An asterisk indicates BMI adjustment for specific phenotype. Phenotype abbreviations: type 2 diabetes (T2D), fasting glucose (FG), fasting insulin (FI), glycated haemoglobin $(\mathrm{HbA1}$ ), fasting pro-insulin (FPI), insulin sensitivity index (ISI), disposition index (DI), corrected insulin response (CIR), corrected insulin response adjusted for Insulin Sensitivity Index (CIR_ISI), incremental insulin at 30 min (Incr30), area under the curve of insulin levels during OGTT (AUCIns), insulin response at $30 \mathrm{~min}$ (Ins30), 2 hour Glucose (2hrGlucose), triglycerides (TG), high density lipoprotein cholesterol (HDL-C), low density lipoprotein cholesterol (LDL-C), total cholesterol (TC), hip circumference (HIP), waist circumference (WC), waist to hip ratio (WHR), body mass index (BMI), systolic blood pressure (SBP), diastolic blood pressure (DBP), hypertension (HTN), coronary artery disease (CAD), eGFR creatinine diabetes mellitus (eGFRcrea_DM), eGFR creatinine non diabetes mellitus (eGFRcrea_nonDM), eGFR creatinine all (eGFRcrea_overall), eGFR cystatin C all (eGFRcys_overall), chronic kidney disease (CKD), white blood cell counts (WBC), intercellular adhesion molecule 1 (ICAM-1), C-reactive protein (CRP), plasminogen activator inhibitor 1 (PAI-1), GT glycaemic traits.

Figure 4. Effects of established FG/FI (a,b) loci onPAI-1 levels and HOMA-B

(a)/HOMA-IR (b). The X-axis represents the direction of loci effects on PAI-1, while the Y-axis shows effects $\beta$-cell function and insulin resistance as reflected by HOMAB/-IR, respectively. 


\section{Figure 1.}

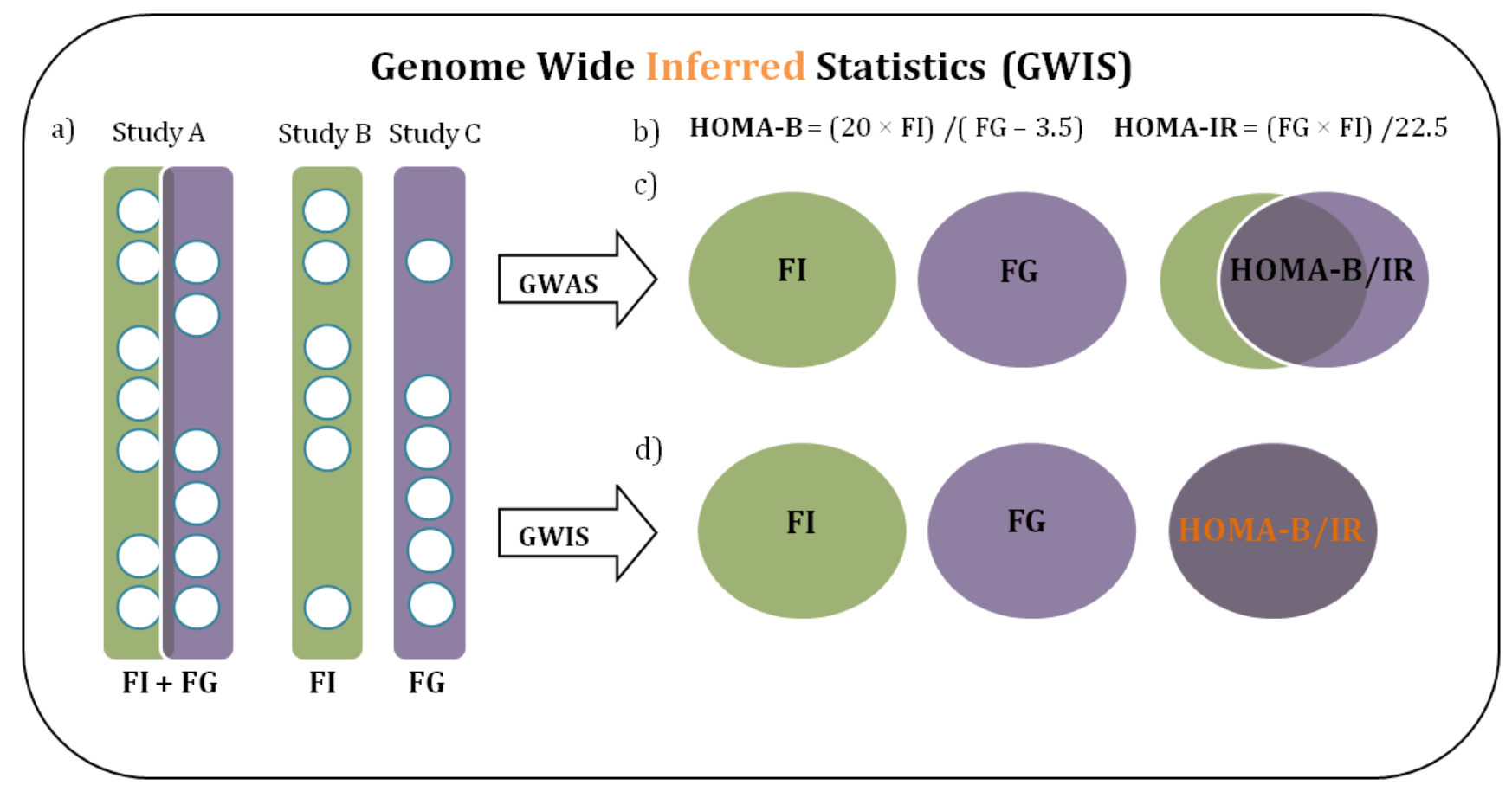


bioRxiv preprint doi: https://doi.org/10.1101/496802; this version posted December 28, 2018. The copyright holder for this preprint (which was not certified by peer review) is the author/funder, who has granted bioRxiv a license to display the preprint in perpetuity. It is made available under aCC-BY-NC-ND 4.0 International license.

\section{Figure 2.}

a) T2D loci, significance of effect on HOMA-B

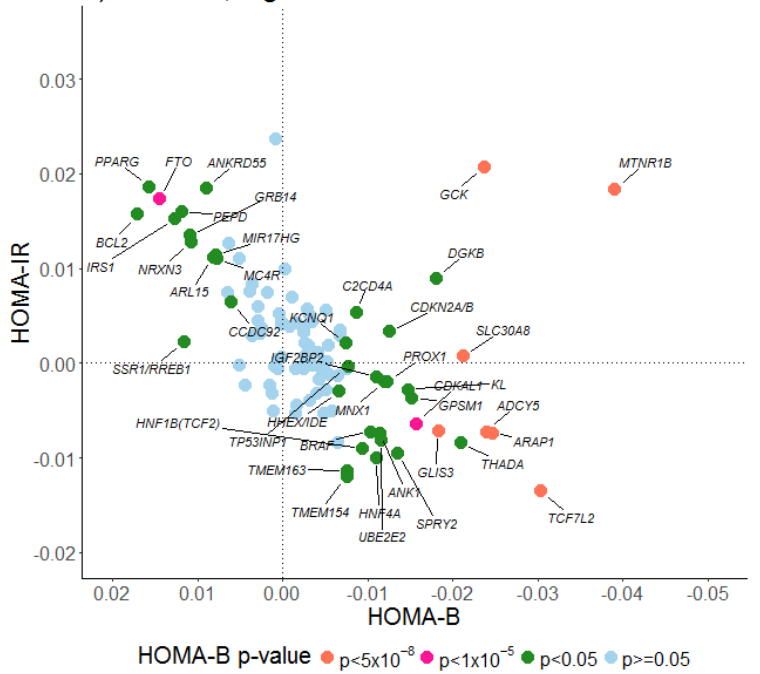

c) FG/FI loci, significance of effect on HOMA-B

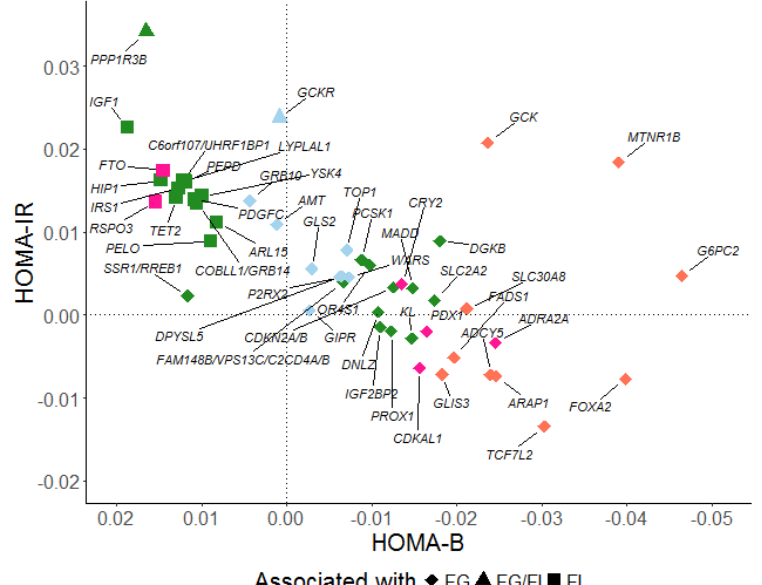

Associated with $\bullet \mathrm{FG} \boldsymbol{A G} / \mathrm{FI} \mathbf{F I}$

HOMA-B p-value $\bullet p<5 \times 10^{-8} \bullet p<1 \times 10^{-5} \bullet p<0.05 \bullet p>=0.05$

e) $\mathrm{HbA} 1 \mathrm{C}$ loci, significance of effect on HOMA-B

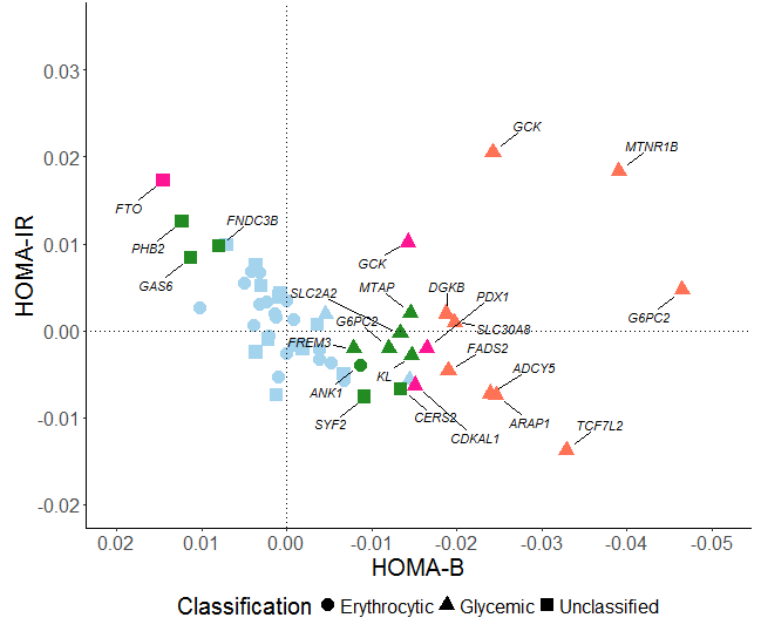

HOMA-B p-value $p<5 \times 10^{-8} \bullet p<1 \times 10^{-5} \bullet p<0.05 \bullet p>=0.05$ b) T2D loci, significance of effect on HOMA-IR

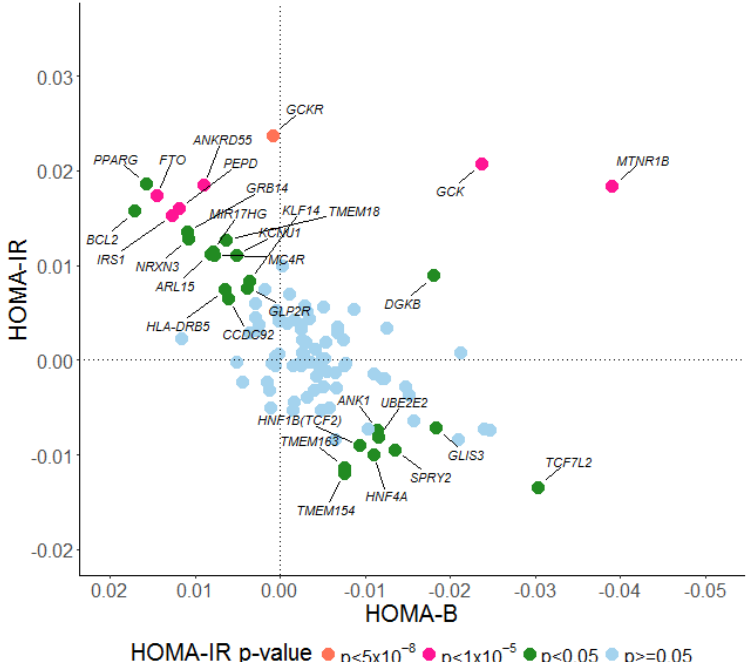

d) FG/FI loci, significance of effect on HOMA-IR

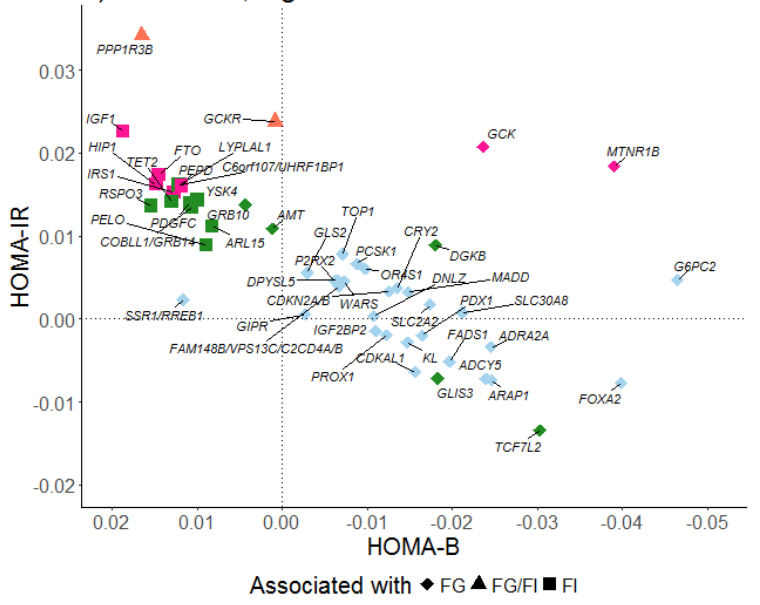

HOMA-IR p-value $\bullet p<5 \times 10^{-8} \bullet p<1 \times 10^{-5} \bullet p<0.05 \bullet p>=0.05$

f) $\mathrm{HbA} 1 \mathrm{C}$ loci, significance of effect on HOMA-IR

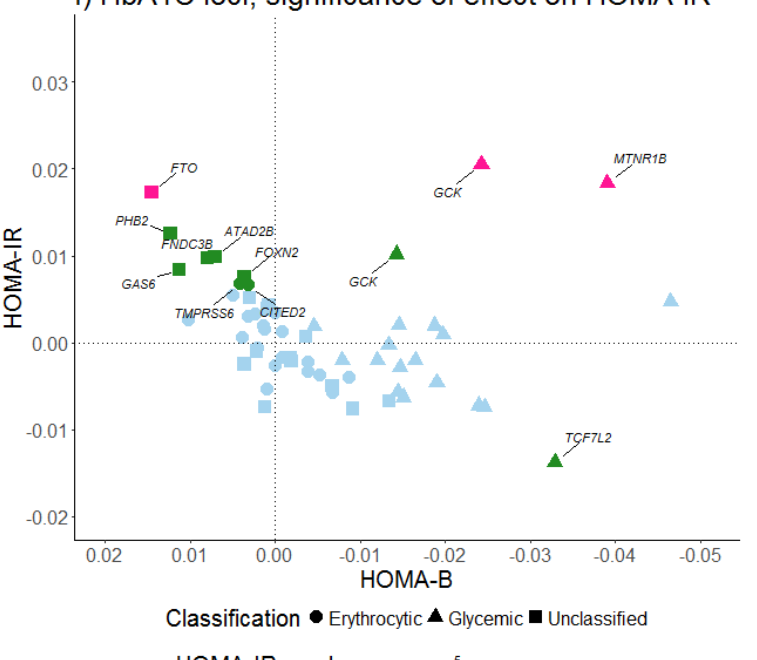

HOMA-IR p-value $\bullet p<1 \times 10^{-5} \bullet p<0.05 \bullet p>=0.05$ 
bioRxiv preprint doi: https://doi.org/10.1101/496802; this version posted December 28, 2018. The copyright holder for this preprint (which was not certified by peer review) is the author/funder, who has granted bioRxiv a license to display the preprint in perpetuity. It is made available under aCC-BY-NC-ND 4.0 International license.

\section{Figure 3.}

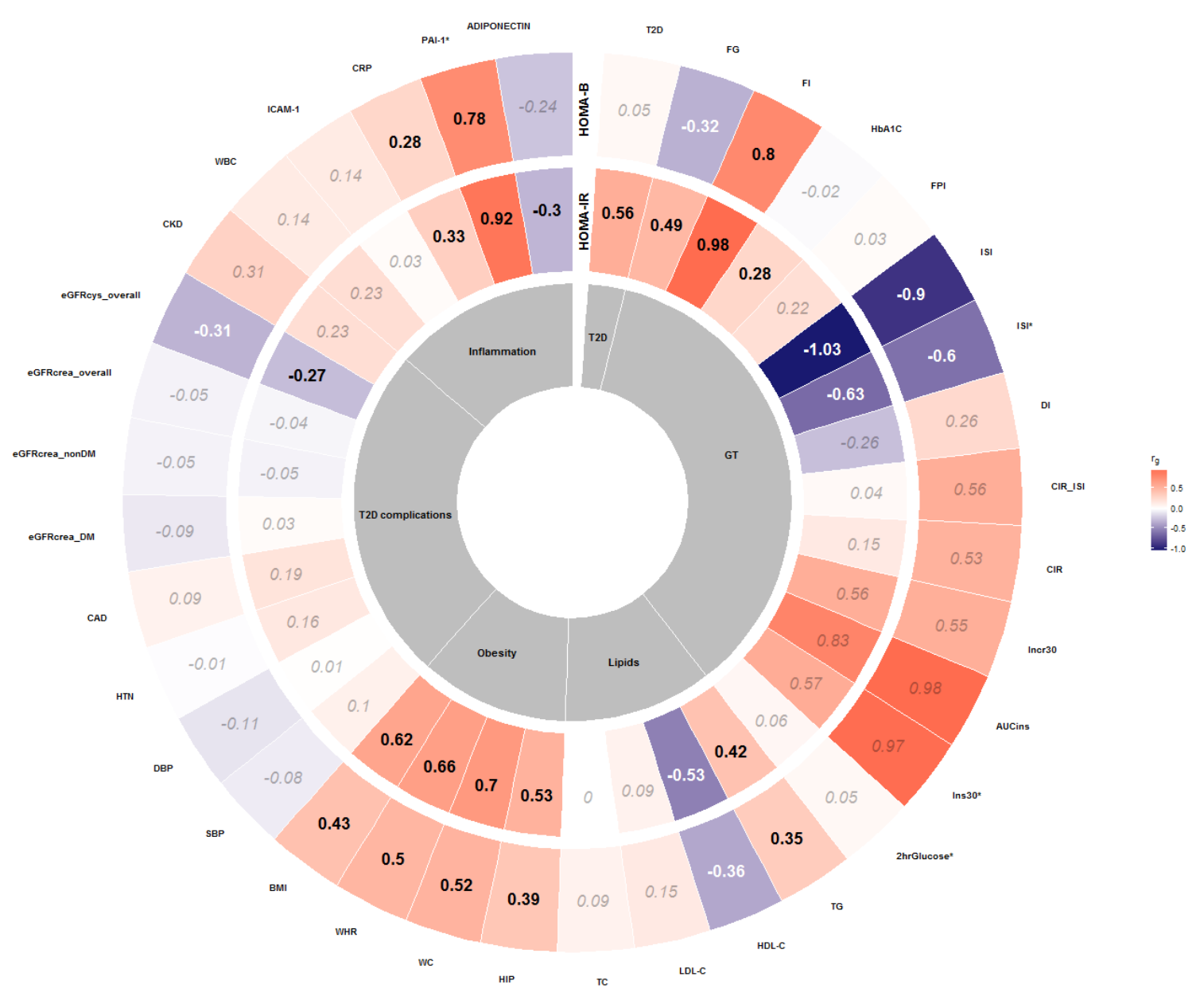


bioRxiv preprint doi: https://doi.org/10.1101/496802; this version posted December 28,2018 . The copyriaht holder for this preprint (which was not certified by peer review) is the author/funder, who has granted bioRxiv a license to display the preprint in perpetuity. It is made available under aCC-BY-NC-ND 4.0 International license.

\section{Figure 4.}

a) FG loci, significance of effect on HOMA-B vs. PAI-1

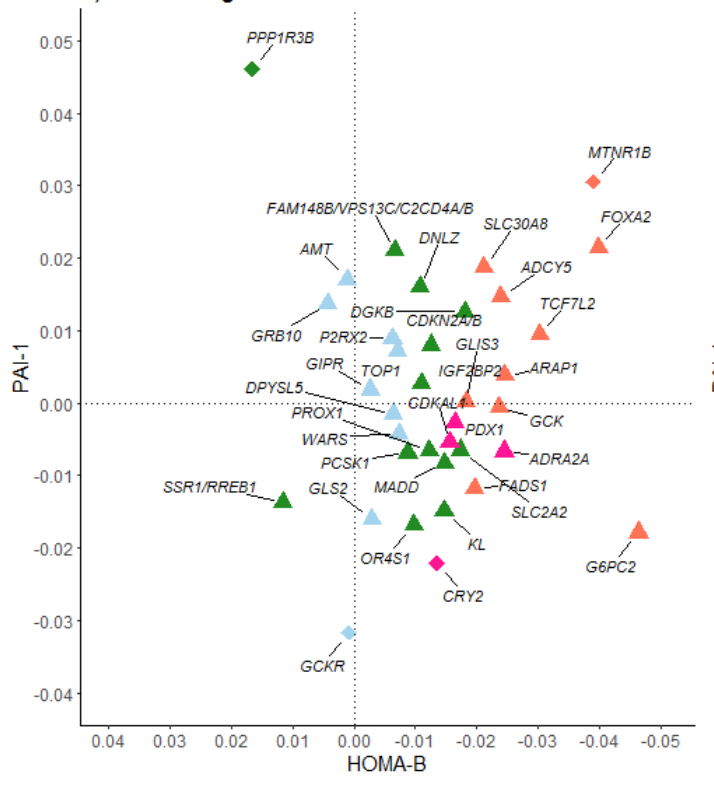

PAl-1 p-value $\bullet p<0.05 \boldsymbol{\Delta}_{\mathrm{p}>=0.05}$ b) FI loci, significance of effect on HOMA-IR vs. PAI-1

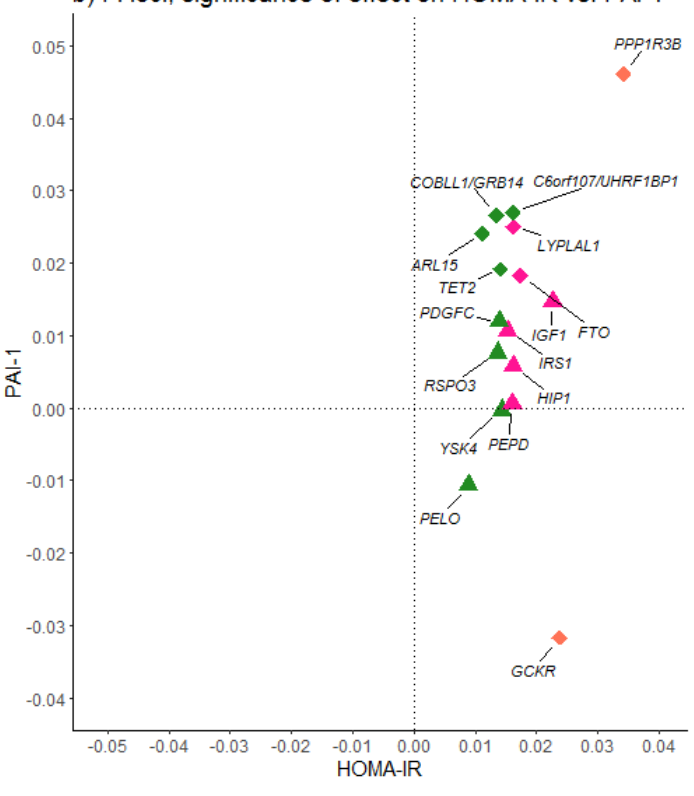

PAl-1 p-value $\bullet p<0.05 \boldsymbol{\Delta}_{p>=0.05}$

HOMA-IR p-value $p<5 \times 10^{-8} \quad p<1 \times 10^{-5} \bullet p<0.05$ 